Journal of Animal and Veterinary Advances 10 (8): 993-998, 2011

ISSN: $1680-5593$

(C) Medwell Journals, 2011

\title{
Optimization of Synbiotics and Growth Factors for Calcium Conversion of Lactobacillus rhamnosus Based on Response Surface Methodology
}

\author{
A.P. Luo, Y.Y. Tang, Y.Y. Yin and L.F. Wang \\ College of Life Science, Guizhou University, Guiyang, Guizhou Province, P.R. China
}

\begin{abstract}
To optimize prebiotics and growth factors for maximizing the calcium conversion of a probiotic, Lactobacillus rhamnosus during the fermentation of boving bone-meal and to assess the effects of these factors by using response surface methodology. After single factor experiment determined the addition of prebiotics and growth factors, conversion rate characteristic of calcium as indicators. Response Surface Methodology (RSM) was used to optimize the best for enhancing the prebiotics and growth factors of Lactobacillus rhamnosus for calcium conversion. It was found that prebiotics were the most effective for calcium conversion, amino acids second, vitamin minimum the last. Analyzing the response surface contour and surface plots, the optimal combination of the prebiotics and growth factors were determined as nicotinic $\mathrm{acid} / \mathrm{vitamin} \mathrm{B}_{2}=2.82: 1$, arginine/met $=1.82: 1$, glucose $/$ isomaltooligosaccharide $=3.80: 1$. The best conversion rate of calcium was $28.32 \%$.
\end{abstract}

Key words: Lactobacillus rhamnosus, probiotic, response surface methodology, synbiotics, calcium formutions, China

\section{INTRODUCTION}

Synbiotics, a combination of probiotics and prebiotics can induce additional effects (Katharina et al., 2007). A positive outcome of prebiotics is promoted by a high level for calcium (Rachmilewitz et al., 2004). A synbiotic is a product containing prebiotics and probiotics and in which the prebiotic compound selectively favors the probiotic compound (Schrezenmeir and Vrese, 2001). Lactobacillus rhamnosus, originally isolated from human intestinal tract has been proven as an important probiotics functioned in the human body (Zhang et al., 2000).

L. rhamnosus have complex nutritional requirements and are strictly fermentative. They were regarded with the capacity of intestinal cell lines, host immunostimulation (Kuisma et al., 2003). L. rhamnosus are potential Llactate due to their homofermenting capacity (Begovic et al., 2009).

They lacks an enzyme to self-synthesize vitamins and amino acids (Fengjie et al., 2002). Therefore, productivity could be significantly influenced by growth nutrients, especially synbiotics and growth factor.

The study was undertaken bovine bone-meal as fermentation substrate used the technologies of microbial fermentation combined with optimization of important prebiotics and growth factors for Lactobacillus rhamnosus are needed to promote calcium transformation and to determine the optimal production of L. rhamnosus by response surface methodology (Azaola et al.,1999).

\section{MATERIALS AND METHODS}

Bovine bone was obtained from supermaker in Gui Yang. All other chemicals were of reagent grade.

Strains and culture conditions: Lactobacillus rhamnosus 1.2466 was provided by the Institute of Microbiology, Chinese Academy of Science. Strain of $L$. rhamnosus was activated in deMan-Rogosa-Sharpe (MRS) broth at $37 \pm 1^{\circ} \mathrm{C}$ for $24 \mathrm{~h}$ determined as viablecount after two generation microecological additives could reach up to $10^{6} \mathrm{cfu} \mathrm{\textrm {mL } ^ { - 1 }}$ then inoculated with $3 \%(\mathrm{w} / \mathrm{v})$ to the formula of fermentation: bovine bone meal $15 \%$, glucose $5 \%$, skim milk $10 \%$, MRS culture medium $2 \%$, distilled water $68 \%$ medium $\mathrm{pH} 6.2$ at $37^{\circ} \mathrm{C} 100 \mathrm{r} \mathrm{min}^{-1}$ for $24 \mathrm{~h}$ microecological additives could reach up to $10^{6} \mathrm{cfu} \mathrm{mL}^{-1}$ then inoculated to the optimum formula of the expanded fermentation: bovine bone meal $4.45 \%$, glucose $12.26 \%$, growth factor, distilled water, pH 6.2 (Yin et al., 2009a, b).

Single factor experiment: $L$. rhamnosus have complex nutritional requirements and are strictly fermentative based on MRS broth, addtioned different kinds of prebiotics and growth factors, the controlled trials were as follows:

Corresponding Author: Y.Y. Tang, College of Life Science, Guizhou University, Guiyang, Guizhou Province, P.R. China 
- Control group: MRS medium

- Trial $\chi:$ (vitamin trials)

- Trial $^{-}$: MRS medium+0.02\% vitamin $B_{1}$

- Trisal $\alpha$ : MRS medium+0.02\% vitamin $B_{2}$

- Trisal $\beta$ : MRS medium $+0.06 \%$ vitamin $\mathrm{B}_{6}$

- Trisal $\chi$ : MRS medium+0.06\% vitamin PP

- Trisal $\delta$ - $\phi$ : (Amino acid trials)

- Trisal $\delta$ : MRS medium $+0.5 \%$ arginine

- Trisale: MRS medium $+0.5 \%$ methionine

- Trisal $\phi$ : MRS medium $+1.0 \%$ cysteine

- Trisaly- $\varphi$ : Prebiotics trials (Wei et al., 2007)

- Trisal $\chi$ : MRS medium $+2.0 \%$ inulin

- Trisal $\eta$ : MRS medium $+2.0 \%$ isomaltooligosaccharide

- Trisall: MRS medium+2.0\% fructo-oligosaccharides

- Trisal $\varphi$ : MRS medium $+2.0 \%$ chitosan

An inoculum size of $3 \%(\mathrm{v} / \mathrm{v})$ L. rhamnosus to MRS medium, fermented for $24 \mathrm{~h}$ determined as colony forming units as indicator selectived the addtion of prebiotics and growth factors.

Response surface methodology: Optimization the selected prebiotics and growth factors for L. rhamnosus fermenting of boving bone-meal by using response surface methodology of 3 factors 3 -levels in the same time, calcium as indicators.

Statistical analyses: For statistics purpose, part of the experiments were repeated at least three times. All the data were analyzed with the aid of the statistics program Design expert 7.0.

\section{RESULTS AND DISCUSSION}

The experiments were carried out according to the experimental plan understand the influence of prebiotics and growth factors on L. rhamnosus shown in Table 1. It was evident that the viablecount of trial $\alpha$, trial $\chi$ and trial $\varepsilon$ were obtained at $1.2 \times 10^{9}, 6.0 \times 10^{9}, 3.3 \times 10^{9} \mathrm{cfu} \mathrm{mL}^{-1}$ after fermentation. The trials were significantly different $(p<0.05)$ between control group that vitamin $B_{2}$, vitamin $P P$ and methionine promoted in growth for LGG. The groups on $\bar{\beta}, \phi$ and $\varphi$ were significantly lower than control group and significantly different $(\mathrm{p}<0.05)$.

It was evident that the vitamin $B_{1}$, vitamin $B_{6}$, cysteine and chitosan inhibiting the growth for LGG. For this study, vitamin $B_{1}$, vitamin $B_{6}$, cysteine and chitosan was not selected. The results from the 1st experiment on prebiotics about the affect of calcium conversation are shown in Fig. 1. It can be concluded that the addition of $2.0 \sim 8.0 \%$ for isomaltooligosaccharide and Fructooligosaccharides may benefit the conversation of calcium. When treated at $8 \%$, the conversastion reach to maximum. With an increase in go down. Similarly with the addition

\begin{tabular}{|c|c|c|c|c|c|c|}
\hline $\begin{array}{l}\text { Trial } \\
\text { group }\end{array}$ & Control group & - & $\alpha$ & $\beta$ & $x$ & 8 \\
\hline LGG & $0.6 \pm 0.05^{c}$ & $0.2 \pm 0.13^{\mathrm{a}}$ & $1.2 \pm 0.20^{\mathrm{d}}$ & $0.4 \pm 0.03^{b}$ & $0.6 \pm 0.03^{f}$ & $0.8 \pm 0.04^{b}$ \\
\hline $\begin{array}{l}\text { Trial } \\
\text { group }\end{array}$ & $\varepsilon$ & $\phi$ & $\gamma$ & $\eta$ & l & $\varphi$ \\
\hline LGG & $3.3 \pm 0.01^{\mathrm{e}}$ & $0.2 \pm 0.01^{\text {sb }}$ & $0.7 \pm 0.02^{\mathrm{cd}}$ & $0.9 \pm 0.05^{\mathrm{bc}}$ & $0.7 \pm 0.03^{\mathrm{cd}}$ & $0.1 \pm 0.12^{\mathrm{s}}$ \\
\hline
\end{tabular}

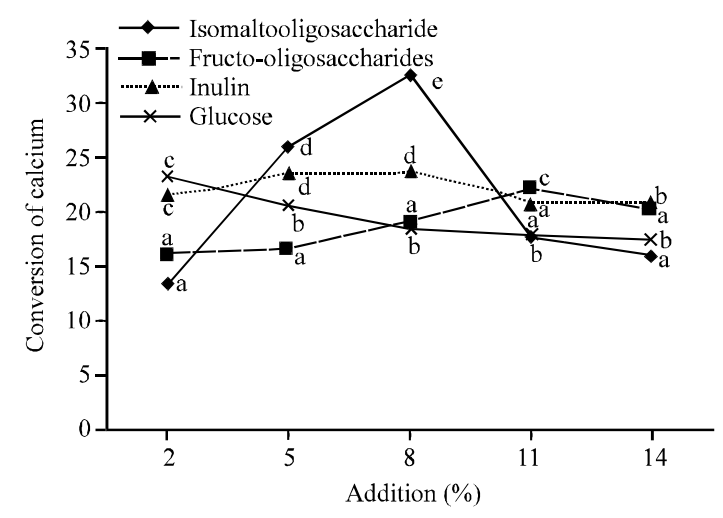

Fig. 1: Effects of different prebiotics LGG fermentation of cattle bone meal

of $2.0 \sim 11.0 \%$ for inulin, the conversation of calcium raising and the maximum for $11 \%$ with an increase in the conversation go down (Galdeano and Perdigon, 2004). This results in prebiotics of isomaltooligosaccharide, Fructo-oligosaccharides and inulin with fit concentration was promotion for L. rhamnosus, excessive addition could control the growth.

Increasing addition of glucose, the conversation of calcium reduced (Lee and Puong, 2002). Therefore, 8\% appeared to isomaltooligosaccharide and Fructooligosaccharides be the optimal addition, inulin were suitable for $11 \%$, glucose $2 \%$. Selection of the compound of isomaltooligosaccharide abd glucose for the next step was caused by the conversation of isomaltooligosaccharide significant higher than control. Therefore, researchers tested the conversation of calcium of standards $(0,20,40,60,80$ and 100\%) for glucose: (glucose + isomaltooligosaccharide) wih the total addition for sugars on $11 \%$.

The single factors experimentations shown in Fig. 1. In the range of $0-80 \%$ was detected that the conversation of calcium presented an increasing tendency with adding glucose. The highest conversationg of $36.65 \%$ was obtained at the addition of $80 \%$ for glucosein the range of $80-100 \%$, the conversation went down quickly. Remarkably, conversation with compoud of glucose and is omaltooligosaccharide at the addition of $80 \%$ higher than other groups. The highest conversationg of glucose/ isomaltooligosaccharide $=4: 1$ (Fig. 2). Vitamin group was selected for vitamin $\mathrm{PP}$ and vitamin $\mathrm{B}_{2}$ and the content was $0.08 \%$ of total addition. Fermentation of zymotic fluid 


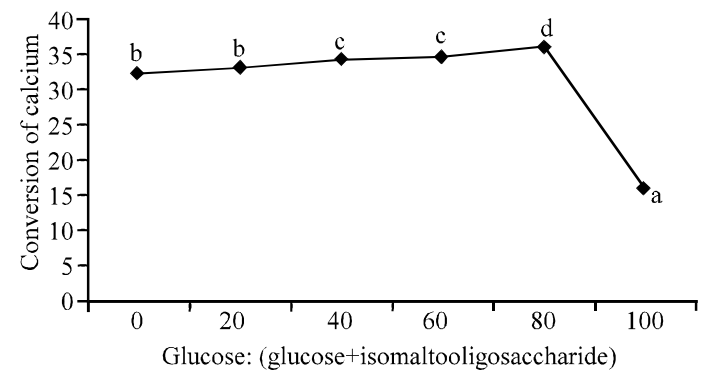

Fig. 2: LGG fermented prebiotics on the impact of cattle bone meal

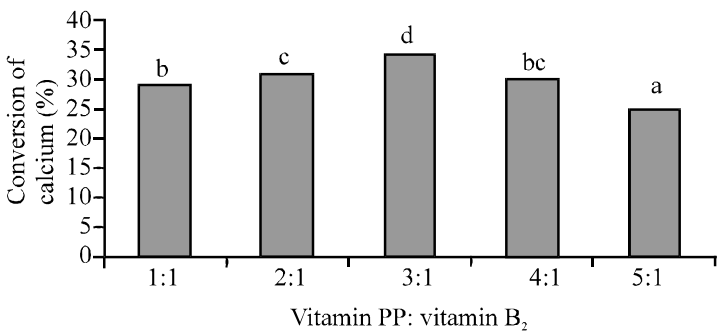

Fig. 3: Vitamin LGG fermented cattle bone meal on the effect on fermented cattle bone meal

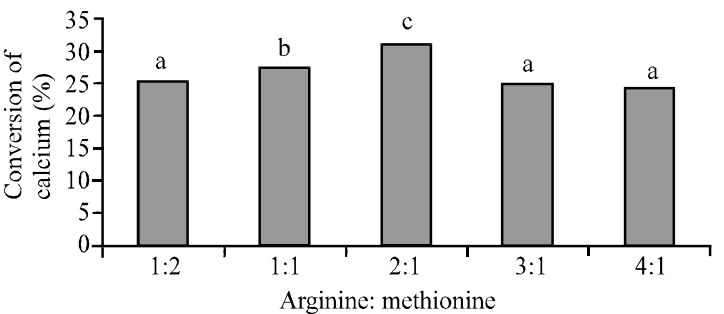

Fig. 4: Amino acids LGG fermented cattle bone meal on the effects

with an concentration boving of $3 \%(\mathrm{w} / \mathrm{v})$ for $72 \mathrm{~h}$ was conducted at 5-level with $1: 1,2: 1,3: 1,4: 1$ and $5: 1$ of vitamin $\mathrm{PP} /$ vitamin $\mathrm{B}_{2}$ (Fig. 3) (Wei et al., 2002). As shown in Fig. 3, the conversation of calcium reached the highest of $33.94 \%$ at vitamin $\mathrm{PP} / \mathrm{vitamin} \mathrm{B}_{2}=3: 1$. The performance of amino acid was selected for arginine and methionine with the proportion at 5 level with $1: 2,1: 1,2: 1,3: 1$ and $4: 1$ of arginine/methionine based on the content $1.0 \%$ of total addition. Conversion rate characteristic of calcium as indicators of arginine/methionine as shown in Fig. 4, the conversation of calcium reached the highest of $30.41 \%$ at arginine $/$ methionine $=2: 1$ and significantly different $(\mathrm{p}<0.05)$.

Optimization using RSM: RSM was used to analyze the experimental data. Optimization, the selected prebiotics and growth factors for L. rhamnosus, fermenting of
Table 2: LGG factors and the level of response surface analysis table

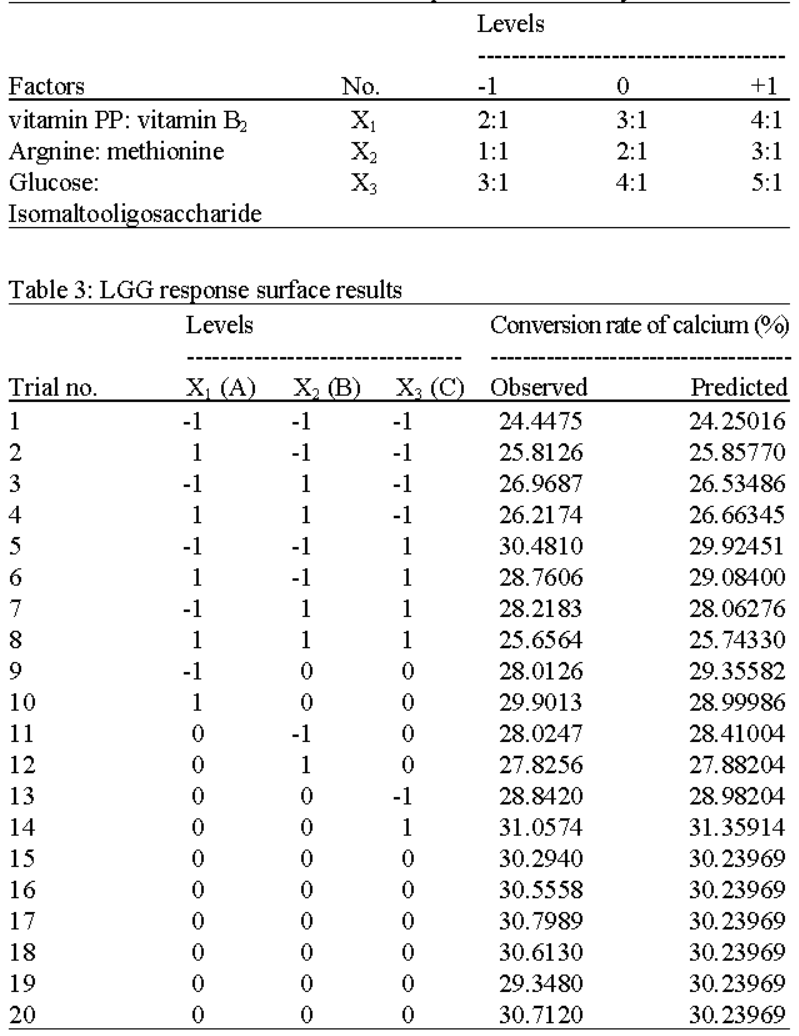

boving bone-meal by using response surface methodology of 3 factors 3-levels in the same time calcium as indicators. An inoculum size of $3 \%(\mathrm{v} / \mathrm{v})$, fermented for $24 \mathrm{~h}$ concentration of boving bone-meal with $5 \%$. The objective of the present study was to find the optimum combination of vitaminPP/B $/ \mathrm{B}_{2}$ and argnine/methionine and glucose/isomaltooligosaccharide to maximize the cell viability of L. rhamonosus for calcium conversion. The experiments were carried out according to the experimental plan shown in Table 2 and 3. The experimental and predicted responses for calcium conversion are shown in Table 3.

Researchers attempted to fit the response variable to a quadratic model in order to correlate the response variable to the independent variables. The behavior of the system was explained by the following quadratic polynomial equation:

$$
\begin{gathered}
Y=30.24-0.18 \mathrm{X}_{1}-0.26 \mathrm{X}_{2}+1.19 \mathrm{X}_{3}-1.06 \mathrm{X}_{12}-2.09 \mathrm{X}_{22^{-}} \\
0.069 \mathrm{X}_{32}-0.37 \mathrm{X}_{1} \mathrm{X}_{2}-0.61 \mathrm{X}_{1} \mathrm{X}_{3}-1.04 \mathrm{X}_{2} \mathrm{X}_{3}
\end{gathered}
$$

The optimum levels of the selected variables were obtained by solving the regression equation and also by analyzing the response surface contour and surface plots. The statistical significance of the model was determined by $F$ test and the analysis of variance for the fitted 
Table 4: Variance of RSD experiments

\begin{tabular}{|c|c|c|c|c|c|c|}
\hline Source & Sum of squares & $\mathrm{df}$ & Mean square & F value & Prob $>F$ & Significance \\
\hline Model & 70.052360 & 9 & 7.783596 & 14.613040 & 0.0001 & *** \\
\hline $\mathrm{X}_{1}$ & 0.316769 & 1 & 0.316769 & 0.594706 & 0.4584 & \\
\hline $\mathrm{X}_{2}$ & 0.696960 & 1 & 0.696960 & 1.308483 & 0.2793 & \\
\hline$X_{3}$ & 14.126510 & 1 & 14.126510 & 26.521320 & 0.0004 & $* *$ \\
\hline $\mathrm{X}_{12}$ & 3.100695 & 1 & 3.100695 & 5.821290 & 0.0365 & $*$ \\
\hline $\mathrm{X}_{22}$ & 12.054270 & 1 & 12.054270 & 22.630860 & 0.0008 & $* *$ \\
\hline $\mathrm{x}_{32}$ & 0.013131 & 1 & 0.013131 & 0.024652 & 0.8784 & $* *$ \\
\hline $\mathrm{X}_{1} \mathrm{X}_{2}$ & 1.093647 & 1 & 1.093647 & 2.053228 & 0.1824 & \\
\hline $\mathrm{X}_{1} \mathrm{X}_{3}$ & 2.996474 & 1 & 2.996474 & 5.625625 & 0.0392 & $*$ \\
\hline $\mathrm{X}_{2} \mathrm{X}_{3}$ & 8.596524 & 1 & 8.596524 & 16.139240 & 0.0024 & $* *$ \\
\hline Residule & 5.326474 & 10 & 0.532647 & - & - & - \\
\hline Lack of fit & 3.883448 & 5 & 0.776690 & 2.691183 & 0.1506 & - \\
\hline Pure error & 1.443026 & 5 & 0.288605 & - & - & - \\
\hline Cor total & 75.378830 & 19 & - & - & - & - \\
\hline
\end{tabular}

quadratic polynomial model is shown in Table 4. The value of $P_{\text {model }}>F$ was $<0.0001$ indicating that the model was significant at the probability level of $\mathrm{a}=0.01$. However, the lack of fit was observed to be insignificant (Plack of fit $>\mathrm{F}=0.1506$ ) implying that the obtained model was adequate to represent the experimental data.

A high value of the correlation coefficient $(\mathrm{R}=$ 0.9293 ) indicating that $92.93 \%$ of the variability in the response could be explained by the secondorder polynomial prediction equation given below indicated a good agreement between the experimental and predicted values of cell viability thus suggesting a high significance for the model. The significance of each coefficient of the model was determined by $\mathrm{F}$ test, this meant that $\mathrm{X}_{3}, \mathrm{X}_{22}$, $\mathrm{X}_{32}, \mathrm{X}_{2} \mathrm{X}_{3}$ were highly significant $(\mathrm{p}<0.001)$ and the $\mathrm{X}_{12}$, $\mathrm{X}_{1} \mathrm{X}_{3}$ were significant $(0.01<\mathrm{p}<0.05) . \mathrm{X}_{1}, \mathrm{X}_{2}, \mathrm{X}_{1} \mathrm{X}_{2}$ were insignificant $(p>0.05)$ that every factors may be the highly significant.

The factors on respond value was $\mathrm{X}_{3}>\mathrm{X}_{2}>\mathrm{X}_{1}$ indicating glucose/isomaltooligosaccharide may be the most important protective agent influencing the resistance of L. rhamonosus to calcium conversion, argnine/ methionine as follow vitamin $\mathrm{PP} / \mathrm{B}_{2}$ was the lowest.

The isoresponse contour and surface plots of RSM as a function of two factors at a time holding all other factors at fixed level (zero for instance) are helpful for understanding both the main and the interaction effects of these two factors (Liu et al., 2003).

The response values for the variables can be predicted from these plots. Figure 5-7 represent the isoresponse contour and surface plots for conversation of calcium (\%) of L. rhamnosus.

The effect of varying concentration of amino acid and vitamin on the conversation of calcium of $L$. rhamnosus while other two viariables were fixed at central concentration is shown in Table 4. So, the interaction between the amion acid and vitamin was insignificant.
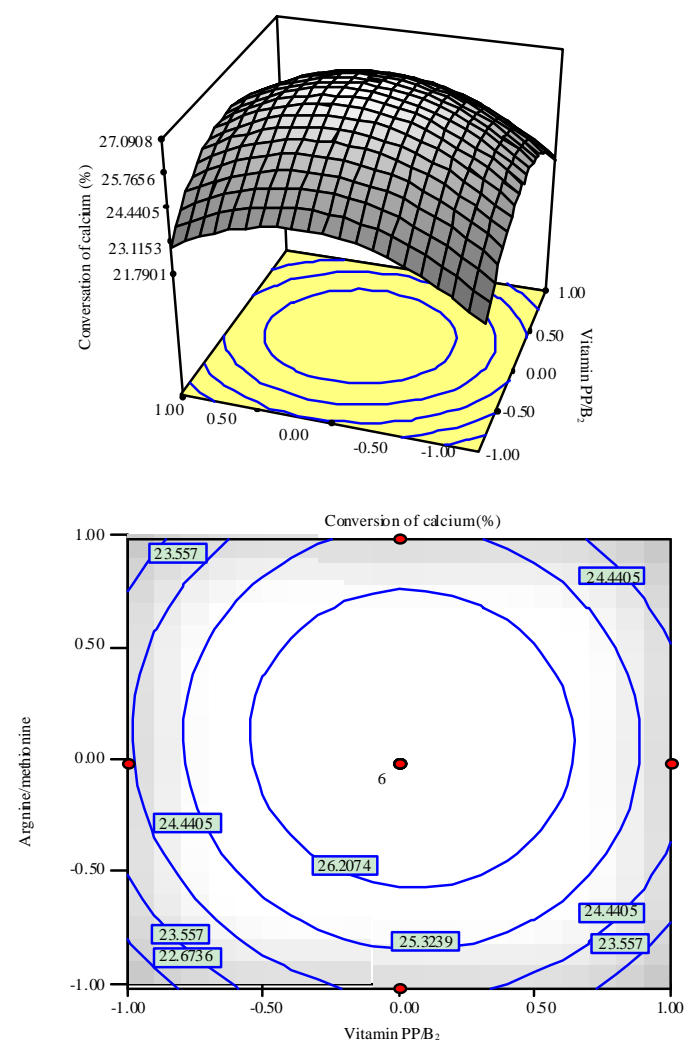

Fig. 5: Effects of conversion rate of calcium on interaction of vitamin and amino acid

Holding amino acid factors at zero level, researchers can know interaction effects on witamin and prebiotica for the conversation of calcium polynomial equation:

$$
\mathrm{Y}=33.896-2.62 \mathrm{X}_{1}-4.42 \mathrm{X}_{2}-1.06 \mathrm{X}_{12}-0.37 \mathrm{X}_{1} \mathrm{X}_{2}-2.09 \mathrm{X}_{22}
$$

The effect of varying concentration of prebiotics and vitamin on the conversation of calcium of L. rhamnosus is shown in Table 4. 

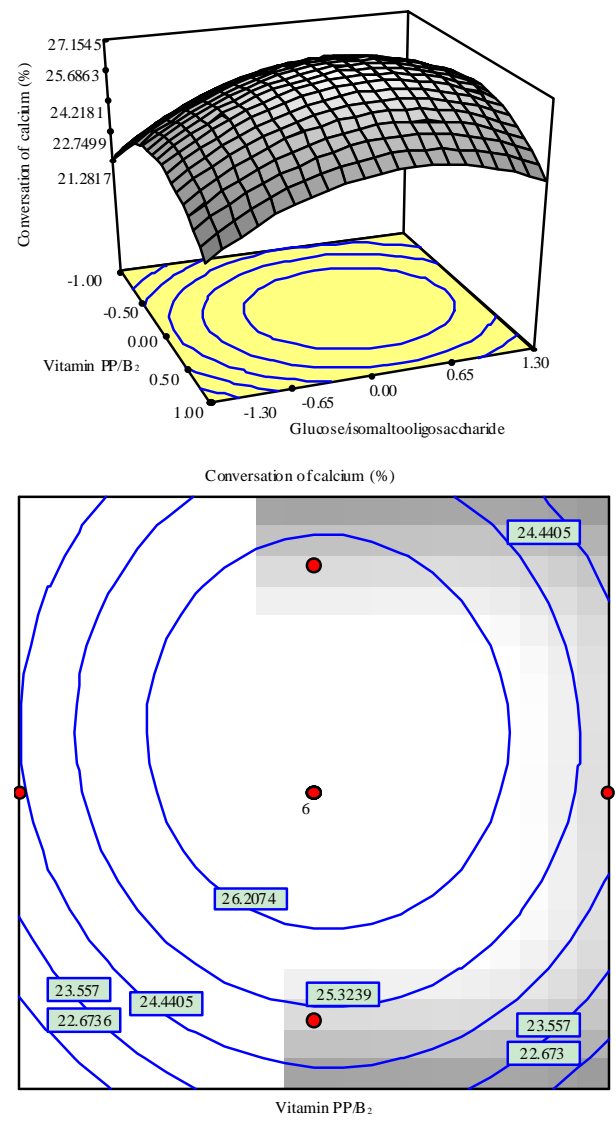

Fig. 6: Effects of conversion rate of calcium on the interaction of vitamins and prebiotics

So, the interaction between the prebiotics and vitamin was significant. Holding vitimin factors at zero level, researchers can know interaction effects on vitamin and prebiotica for the conversation of calcium polynomial equation:

$$
\mathrm{Y}=21.36-1.4 \mathrm{X}_{1}-0.89 \mathrm{X}_{3}-1.06 \mathrm{X}_{12}-0.069 \mathrm{X}_{32}-0.61 \mathrm{X}_{1} \mathrm{X}_{3}
$$

The effect of varying concentration of prebiotics and amino acid on the conversation of calcium of L. rhamnosus is shown in Table 4. So, the interaction between the prebiotics and amino acid was significant. Holding vitimin factors at zero level, researchers can know interaction effects on amino acid and prebiotica for the conversation of calcium polynomial equation:

$$
\mathrm{Y}=20.16-1.37 \mathrm{X}_{2}-0.64 \mathrm{X}_{3}-2.09 \mathrm{X}_{22}-0.069 \mathrm{X}_{32}-1.04 \mathrm{X}_{2} \mathrm{X}_{3}
$$

From equations derived by differentiating equations, the optimum values for the independent variables investigated were nicotinic acid/vitamin $\mathrm{B}_{2}=2.82: 1$,
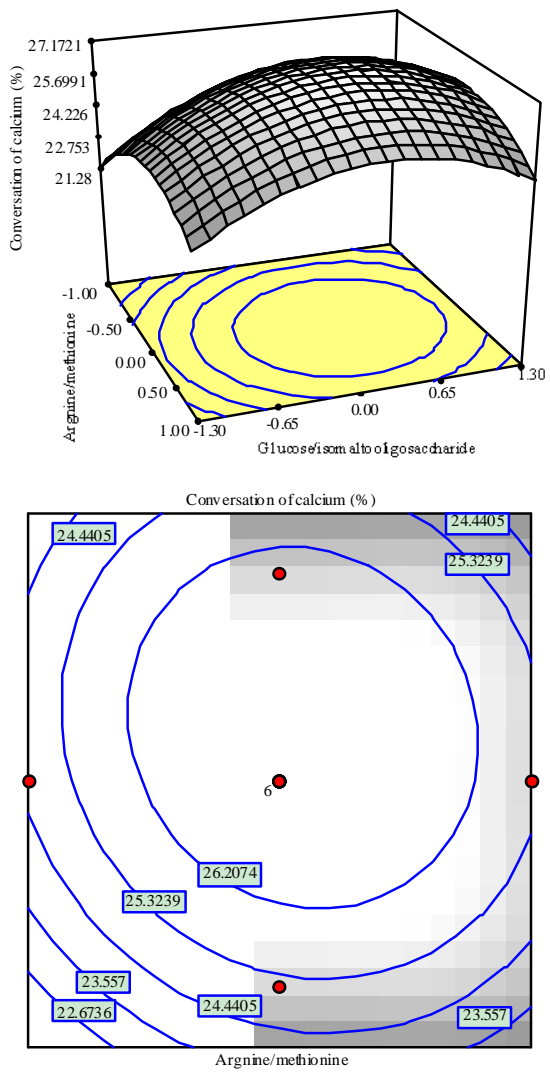

Fig. 7: Effects of conversion rate of calcium on the interaction of amino acids and glycogen

arginine $/$ met $=1.82: 1$, glucose $/$ isomaltooligosaccharide $=$ $3.80: 1$. The best conversion rate of calcium was $28.32 \%$. To confirm the results, conversation of calcium in this optimum protective medium and a viability of $96.05 \%$ (theory conversation of calcium was $27.55 \%$ ). The good correlation between these results verified the goodness of fit of the model.

\section{CONCLUSION}

Analyzing the response surface contour and surface plots, prebiotics were the most effective for calcium conversion, amino acids second and vitamin minimum the last. The optimal combination of the prebiotics and growth factors were determined as nicotinic acid: vitamin $\mathrm{B}_{2}=2.82: 1$, arginine: met $=1.82: 1$, glucose: isomaltooligosaccharide $=3.80: 1$. The best conversion rate of calcium was $28.32 \%$.

\section{ACKNOWLEDGEMENTS}

This research was financially supported by the Support Project of Science and Technology of Guizhou 
province of the People's Republic of China (GZNY NO.: [2007] 3021) and Guizhou technical system of modem beef industry (GZCYTX-0301-03).

\section{REFERENCES}

Azaola, A., P. Bustarnante, S. Huertu, G. Saucedo, R. Gonzalez, C. Ramos and S. Saval, 1999. Use of response surface methodology to describe biomass production of Bifidobacterium infantis in complex media. Bio. Tech., 13: 93-95.

Begovic, J., D. Fira, A. Terzic-Vidojevic and L. Topisirovic, 2009. Influence of carbohydrates on cell properties of Lactobacillus rhamnosus. Cen. Eur. Biol. J., 5: 103-110.

Fengjie, C., W. Caixia, Y. Li, L. Zhe and G. Rajashekara, 2002. Co-production of Lactic Acid and Lactobacillus rhamnosus Cells from Whey Permeate with Nutrient Supplements. Bio. Tech., 10: 426-427.

Galdeano, C. and G. Perdigon, 2004. Role of viability of probiotic strains in their persistence in the gut and in mucosal immune stimulation. J. Applied Microb., 97: 673-681.

Katharina, E.S., P. Ade, B. Marten, P. Weber and W. Timm et al., 2007. Prebiotics, probiotics and synbiotics affect mineral absorption, bone mineral content and bone structure. J. Nutr., 137: 838-846.

Kuisma, J., S. Mentula, H. Jarvinen, A. Kahri, M. Saxelin and M. Farkkila, 2003. Effect of L. rhamnosus GG on ileal pouch inflammation and microbial flora. Aliment. Pharmacol. Ther., 17: 509-515.

Lee, Y.K. and K.Y. Puong, 2002. Competition for adhesion between probiotics and human gastrointestinal pathogens in the presence of carbohydrate. Br. J. Nutr., 88: 101-108.
Liu, J.Z., L.P. Weng, Q.L. Zhang, H. Xu and L.M. Ji, 2003. Optimization of glucose oxidase production by Aspergillus niger in a benchtopbioreactor using response surface methodology. World J. Microb. Biot., 19: 317-323.

Rachmilewitz, D., K. Katakura, F. Karmeli, T. Hayashi and C. Reinus et al., 2004. Toll-like receptor 9 signaling mediates the anti-inflammatory effects of probiotics in murine experimental colitis. Gastroenterol., 126: 520-528.

Schrezenmeir, J. and M. Vrese, 2001. Probiotics, prebiotics and synbiotics-approaching a definition. Am. J. Clin. Nutr., 73: $361-364$.

Wei, H., V. Loimaranta, J. Tenovuo, S. Rokka and E.L. Syvaoja et al., 2002. Stability and activity of specific antibodies against Streptococcus mutans and Streptococcus sobrinus in bovine milk fermented with $L$. rhamnosus strain GG or treated at ultra-high temperature. Oral Microb. Immunol., 17: 9-15.

Wei, H., Y. Xu, Y.H. Xiong and F. Xu, 2007. Synergistic antidigestion effect of Lactobacillus rhamnosus and bovine colostrums in simulated gastrointestinal tract in vitro. Appllied Microb. Biot., 75: 619-626.

Yin, Y.Y., A.P. Luo, J.T. Wu and L. Yu, 2009a. Comparative study on calcium transformation in cattle bone powder by fermentation of Lctobacillus rhamnosus GG and Bifidobacterium infantis. Food Sci., 9: 144-149.

Yin, Y.Y., A.P Luo, S. Li and T.T. Wan, $2009 \mathrm{~b}$. Optimization of Symbiotic Fermentation of Lactobacillus acidophilus and Lactobacillus rhamnosus for Calcium Transformation in Bovine Bone Powder. Food Sci., 21: 178-183.

Zhang, W., J.H. Kim, C.M. Franco and A.P. Middelberg, 2000. Characterisation of the shrinkage of calcium alginate gel membrane with immobilised Lactobacillus rhamnosus. Applied Microb. Biot., 54: $28-32$. 